\title{
Inner Automorphisms, Discrete and Bäcklund Transformations of Integrable Systems
}

\author{
A. N. Leznov \\ Communicated by Charles Li, received March 25, 2005
}

\begin{abstract}
It is shown that there exist two inner automorphisms and a discrete transformation besides the usual Bäcklund transformation for the integrable focusing nonlinear Schrödinger equation. By virtue of the inner automorphisms, the discrete transformation can generate multi-soliton solutions. The expressions for multi-soliton solutions generated by the discrete and the Bäcklund transformations turn out to be the same.
\end{abstract}

\section{Contents}

1. Introduction

2. Discrete Transformation 150

3. Bäcklund Transformation 151

4. Multisoliton Solutions 153

5. Bäcklund Transformation in Its Original Form 154

6. Second Automorphism $\sigma_{2}$ of the Nonlinear Schrödinger System 154

$\begin{array}{ll}\text { 7. Outlook } & 157\end{array}$

8. Appendix 158

$\begin{array}{ll}\text { References } & 158\end{array}$

\section{Introduction}

In a series of papers by the author starting in the 80's of the previous century [1], a simple method for constructing integrable systems together with their soliton-like solutions was proposed. This method requires only two calculational steps - solving a system of linear algebraic equations and performing a Gaussian decomposition of a polynomial into a product determined by its roots. This leads to a nonlinear symmetry of integrable systems termed a discrete substitution or an integrable

1991 Mathematics Subject Classification. Primary 35 Secondary 78.

Key words and phrases. Inner automorphism, discrete transformation, Bäcklund transformation, integrable system, multi-soliton solution. 
mapping. A discrete substitution is a nonlinear transformation (with no additional parameters) that, given an arbitrary solution of an integrable system, produces a new solution according to certain rules.

All systems of equations invariant with respect to such a mapping are united into a hierarchy of integrable systems that bears the name of the corresponding discrete substitution. This substitution is a canonical transformation $[\mathbf{2}][\mathbf{3}]$ which explains successful applications of methods of canonical transformation theory to integrable systems $[\mathbf{9}]$.

Let us assume that an integrable system has some inner automorphism $\sigma$. This implies that there is a solution that is also invariant under $\sigma$. Usually, such solutions are interesting for applications. In general, a discrete transformation will not commute with $\sigma$. However, it is possible starting from a non-invariant solution to produce a "good" invariant solution after several applications of the discrete transformation (implementation of this scheme is outlined in [4] and [5]).

On the other hand, it is well known that Bäcklund transformations contain additional parameters and therefore allow to increase the number of parameters in the solution after their application. Because the method of discrete substitution allowed to obtain n-soliton solutions directly and explicitly, the author had no interest in Bäcklund transformations from the point of view of obtaining soliton solutions. But Bäcklund transformations have a wider sence and we would like to close this loophole in the present paper.

The goal of the present paper is to show how to construct Bäcklund transformations for integrable systems using methods of [4],[5]. We restrict ourselves to the well-known example of the nonlinear Schrödinger equation to ease the demonstration of the main idea and methods. The extension to the general case is obvious.

\section{Discrete Transformation}

All considerations of the present paper are given for the simplist example of the nonlinear Schrödinger equations. However, it will become clear from our arguments, detailed calculations below, and references that our method is applicable to all integrable systems with soliton-like solutions.

The nonlinear Schrödinger equation can be represented in two forms: the classical one for one complex-valued unknown function $\psi(x, t)$

$$
-2 i \psi_{t}+\psi_{x x}+2(\psi \bar{\psi}) \psi=0,
$$

and as a wider system of two equations for two unknown complex-valued functions functions $u, v$

$$
-i 2 u_{t}+u_{x x}+2(u v) u=0, \quad i 2 v_{t}+v_{x x}+2(u v) v=0 .
$$

(The factor of 2 multiplying time derivatives can be of course eliminated by redefining the time variable.) The last system is obviously invariant under an inner automorphism $\sigma_{1}: u \rightarrow \bar{v}, v \rightarrow \bar{u}$ and thus it has solutions invariant with respect to this change of variables. Such solutions satisfy $u=\bar{v}=\psi$ and are therefore also solutions of (2.1).

The system (2.2) is invariant with respect to the following nonlinear invertible transformation

$$
U=\frac{1}{v}, \quad V=v\left(u v-(\ln v)_{x x}\right), \quad v=\frac{1}{U}, \quad u=U\left(U V-(\ln U)_{x x}\right),
$$


which is referred to as a discrete transformation, discrete substitution or an integrable mapping. Transformation (2.3) takes a given solution $(u, v)$ of the system $(2.2)$ to a new one $(U, V)$. But this transformation is not invariant with respect to $\sigma_{1}$. Thus, if the initial solution has a property $(u=\bar{v})$, the new solution $(U, V)$ will not have this property. Now we make the following very important observation. If we apply the direct discrete transformation $m$ times to a solution of (2.1) $(u=\bar{v})$ with the result $U^{+m}, V^{+m}$ and apply the inverse transformation to the same solution $m$ times with the result $u^{-m}, v^{-m}$, the resulting new solutions are related as follows: $U^{+m}=\bar{u}^{-m}, V^{+m}=\bar{v}^{-m}$. Thus, if we start from an obvious linear solution of the system $(2.2) u_{0}=0,2 i v_{0 t}+v_{0 x x}=0$ and after $2 m$ steps of direct discrete transformation obtain a solution of $2 i u_{2 m t}+u_{2 m x x}=0, v_{2 m}=0$ with $u_{2 m}=\bar{v}_{0}$, we are guaranteed that at the $m$ th step we will obtain a (m-soliton) solution of (2.1) - traditional nonlinear Schrödinger equation. This approach together with the corresponding mathematical formalism is described in details in [4] $[5]$.

\section{Bäcklund Transformation}

Now we would like to find not the symmetry of the enlarged system (2.2) but independently the symmetry of (2.1). This symmetry transformation can contain additional numerical parameters, and after each of its applications, we increase the number of parameters in the solution. This is exactly Bäcklund's original idea. However, one has to keep in mind that, applied to the general solution of the equation, this transformation cannot add any new independent parameters, but can only change the initial functions on which the general solution depends.

Consider a solution of the classical nonlinear Schrödinger equation $\psi=u=\bar{v}$. This equation may be written in Lax pair form

$$
g_{x} g^{-1}=i\left(\begin{array}{rr}
\lambda, & u \\
v & -\lambda
\end{array}\right), \quad g_{t} g^{-1}=i\left(\begin{array}{cc}
\lambda^{2}-\frac{u v}{2}, & \lambda u-i \frac{u_{x}}{2} \\
\lambda v+i \frac{v_{x}}{2} & -\lambda^{2}+\frac{u v}{2}
\end{array}\right) .
$$

Now let us use the formalism of [5] and try to find a new solution of the classical nonlinear Schrödinger equation in the form

$$
G=\left(\begin{array}{lr}
\lambda+A & B \\
C & \lambda+D
\end{array}\right) g \equiv P g
$$

where functions $A, B, C$ and $D$ are determined by imposing the condition that the columns (and rows) of the matrix $G$ are linearly dependent with constant coefficients $\left(c_{1}^{i}, c_{2}^{i}\right)$ at two points of the complex $\lambda$ plane $\lambda_{1,2}$. In the Appendix, we show that this condition is not independent, but follows directly from (3.2). From this condition, we immediately obtain $\operatorname{Det} P=\left(\lambda-\lambda_{1}\right)\left(\lambda-\lambda_{2}\right)$ and a linear system of equations for relating functions $A, B, C, D$ to matrix elements of $g$, parameters $\lambda_{i}$, and vectors $c^{i}$

$$
\begin{aligned}
& \left(\lambda_{1}+A\right)(g c)_{1}^{1}+B(g c)_{2}^{1}=0, \quad\left(\lambda_{2}+A\right)(g c)_{1}^{2}+B(g c)_{2}^{2}=0, \\
& C(g c)_{1}^{1}+\left(\lambda_{1}+D\right)(g c)_{2}^{1}=0, \quad C(g c)_{1}^{2}+\left(\lambda_{1}+D\right)(g c)_{2}^{2}=0 .
\end{aligned}
$$

In particular, we have

$$
\left(\lambda_{1}-\lambda_{2}\right)+B\left(\frac{(g c)_{2}^{1}}{(g c)_{1}^{1}}-\frac{(g c)_{2}^{2}}{(g c)_{1}^{2}}\right)=0, \quad\left(\lambda_{1}-\lambda_{2}\right)+C\left(\frac{(g c)_{1}^{1}}{(g c)_{2}^{1}}-\frac{(g c)_{1}^{2}}{(g c)_{2}^{2}}\right)=0 .
$$


Now let us obtain a new L-A pair. For the matrix element $\left(G_{x} G^{-1}\right)_{11}$ we have (3.6)

$$
\left(G_{x} G^{-1}\right)_{11}=\frac{\operatorname{det}\left(\begin{array}{rr}
A_{x}+(\lambda+A) i \lambda+i B v & B_{x}+(\lambda+A) i u-B i \lambda \\
C & \lambda+D
\end{array}\right)}{\operatorname{det} P}=i \lambda
$$

where we used the fact that the determinants in the numerator and denominator have zeros at the same points. Similarly, we obtain

$$
\left(G_{x} G^{-1}\right)_{12}=\frac{\operatorname{det}\left(\begin{array}{lr}
\lambda+A & B \\
A_{x}+(\lambda+A) i \lambda+B i v & B_{x}+(\lambda+A) i u-B i \lambda
\end{array}\right)}{\operatorname{det} P}=i(u-2 B),
$$

and finally

$$
G_{x} G^{-1}=i\left(\begin{array}{lr}
\lambda, & u-2 B \\
v+2 C & -\lambda
\end{array}\right) .
$$

If we did not demand the condition of complex conjugation $u=v^{*}$ to hold, this would exactly be a Bäcklund transformation for the enlarged nonlinear system (2.2). But if we start from a solution of the nonlinear Schrödinger equation (2.1) $u=v^{*}$ and would like to obtain a solution of the same equation, we have to require, according to the last expression, that $\bar{C}=-B$. To this end, it is necessary to use known explicit expressions (3.5). From L-A representation (3.1), it follows that $g$ can be considered as a unitary matrix $g g^{H}=1$ under the assumption that $\lambda$ is real. This means that $g^{-1}=g^{H}$ or in a matrix form (in what follows $\bar{f} \equiv f^{*}$ )

$$
g(\lambda)_{22}=g^{*}(\lambda)_{11}, \quad g(\lambda)_{12}=-g^{*}(\lambda)_{21},
$$

Taking into account that the matrix elements of $g$ are analytic functions of $\lambda$ (as solutions of a differential equation with coefficients analytic in $\lambda$ ), we conclude that

$$
\begin{array}{cl}
\left(g(\lambda)_{22}\right)^{*}=g\left(\lambda^{*}\right)_{11}, & \left(g(\lambda)_{11}\right)^{*}=g\left(\lambda^{*}\right)_{22}, \\
\left(g(\lambda)_{21}\right)^{*}=-g\left(\lambda^{*}\right)_{12}, & \left(g(\lambda)_{12}\right)^{*}=-g\left(\lambda^{*}\right)_{21} .
\end{array}
$$

Now we can evaluate $\bar{C}$. We have

$$
\frac{(g c)_{1}^{1}}{(g c)_{2}^{1}}=\frac{g\left(\lambda_{1}\right)_{11} c_{1}^{1}+g\left(\lambda_{1}\right)_{12} c_{2}^{1}}{g\left(\lambda_{1}\right)_{21} c_{1}^{1}+g\left(\lambda_{1}\right)_{22} c_{2}^{1}}=\frac{g\left(\lambda_{1}\right)_{11}+g\left(\lambda_{1}\right)_{12} \alpha_{1}}{g\left(\lambda_{1}\right)_{21}+g\left(\lambda_{1}\right)_{22} \alpha_{1}}
$$

where $\alpha_{1}=\frac{c_{2}^{1}}{c_{1}^{1}}$. According to the above formulae for complex conjugation, we have

$$
\left(F\left(\lambda_{1}, \alpha_{1}\right)\right)^{*} \equiv\left(\frac{(g c)_{1}^{1}}{(g c)_{2}^{1}}\right)^{*}=\frac{g\left(\lambda_{1}^{*}\right)_{22}-g\left(\lambda_{1}^{*}\right)_{21} \alpha_{1}^{*}}{-g\left(\lambda_{1}^{*}\right)_{12}+g\left(\lambda_{1}^{*}\right)_{11} \alpha_{1}^{*}}=-\frac{1}{F\left(\lambda_{1}^{*},-\frac{1}{\alpha_{1}^{*}}\right.} .
$$

For further manipulations, let us rewrite (3.5) in terms of $F_{1}, F_{2}$ introduced above $(3.10)$

$\left(\lambda_{1}-\lambda_{2}\right)+C\left(F\left(\lambda_{1}, \alpha_{1}\right)-F\left(\lambda_{2}, \alpha_{2}\right)\right)=0, \quad\left(\lambda_{1}-\lambda_{2}\right)+B\left(\frac{1}{F\left(\lambda_{1}, \alpha_{1}\right)}-\frac{1}{F\left(\lambda_{2}, \alpha_{2}\right)}\right)=0$.

The condition $B=-C^{*}$ together with the conjugation properties of the functions $F$ lead to

$$
\lambda_{1}=\lambda_{2}^{*}, \quad \alpha_{1}^{*}=-\frac{1}{\alpha_{2}} .
$$

Thus if for some solution of (2.1), the corresponding element $g$ is known, a new solution of the same equation different from the initial one, can be constructed by 
the rules of present section. (But do not forget about the comments on this subject at the beginning of this section.)

\section{Multisoliton Solutions}

Now let us apply $n$ times the transformation of the previous section to a certain solution of the nonlinear Shrödinger equation (2.1). Each transformation is defined by two complex parameters $\lambda_{i}, \alpha_{i}$. After $\mathrm{n}$ applications, we have for the corresponding matrix $G_{n}$

$$
G_{n}=\left(\begin{array}{lr}
\lambda+A & B \\
C & \lambda+D
\end{array}\right) G_{n-1}=\left(\begin{array}{lr}
\tilde{P}_{11}^{n} & P_{12}^{n-1} \\
P_{21}^{n-1} & \tilde{P}_{22}^{n}
\end{array}\right) g_{0}
$$

Here the notation $\tilde{P}$ means that the coefficient at the highest degree of the corresponding polynomial is equal to one. $G_{n}$ has zero vectors at $2 n$ points of the $\lambda_{i}, \lambda_{i}^{*}$ plane with the coefficients of proportionalities $\alpha_{i},-\frac{1}{\alpha_{i}^{*}}$. For this reason all coefficients of polynomials of the element $G_{n}$ can be obtained from the linear system of equations

$$
\begin{gathered}
\lambda_{i}^{n}+\sum_{k=0}^{n-1} P_{11}^{k} \lambda_{i}^{k}+\sum_{k=0}^{n-1} F\left(\lambda_{i}, \alpha_{i}\right) P_{12}^{k} \lambda_{i}^{k}=0, \\
\sum_{k=0}^{n-1} F^{-1}\left(\lambda_{i}, \alpha_{i}\right) P_{21}^{k} \lambda_{i}^{k}+\lambda_{i}^{n}+\sum_{k=0}^{n-1} P_{22}^{k} \lambda_{i}^{k}=0,
\end{gathered}
$$

where $P_{i j}^{k}$ are the coefficients at $\lambda^{k}$ in the corresponding polynomial. In connection with Cramers rules, coefficients interesting for further considerations are

$$
\begin{gathered}
P_{12}^{n-1}=-\frac{\operatorname{det}_{2 n}\left(\lambda_{i}^{n-1}, . ., \lambda_{i}, 1 ; \lambda_{i}^{n} ; F_{i} \lambda_{i}^{n-2}, \ldots F_{i} \lambda_{i}, F_{i}\right)}{\operatorname{det}_{2 n}\left(\lambda_{i}^{n-1}, . ., \lambda_{i}, 1 ; F_{i} \lambda_{i}^{n-1}, \ldots F_{i} \lambda_{i}, F_{i}\right)}, \\
P_{21}^{n-1}=-\frac{\operatorname{det}_{2 n}\left(\lambda_{i}^{n} ; F_{i}^{-1} \lambda_{i}^{n-2}, \ldots F_{i}^{-1} \lambda_{i}, F_{i}^{-1} ; \lambda_{i}^{n-1}, . ., \lambda_{i}, 1\right)}{\operatorname{det}_{2 n}\left(F_{i}^{-1} \lambda_{i}^{n-1}, \ldots F_{i}^{-1} \lambda_{i}, F_{i}^{-1} ; \lambda_{i}^{n-1}, . ., \lambda_{i}, 1\right)} .
\end{gathered}
$$

In the last formula, we symbolically wrote the structure of each of $2 n$ lines of the corresponding determinant matrices. In the case of a diagonal initial matrix $g_{0}=\exp i\left(\lambda x+\lambda^{2} t\right) h$, formula (4.1) was presented in [4] without any connection with the Bäcklund transformation of the present paper.

Using absolutely the same technique as in the previous section, we obtain

$$
\left(G_{n}\right)_{x} G_{n}^{-1}=i\left(\begin{array}{lr}
\lambda, & u-2 P_{12}^{n-1} \\
v+2 P_{21}^{n-1} & -\lambda
\end{array}\right) .
$$

Thus, after $\mathrm{n}$ steps of Bäcklund transformations each of which is defined by parameters $\left(\lambda_{i}, \lambda_{i}^{*}, \alpha_{i},-\frac{1}{\alpha_{i}^{*}}\right)$, we get a new solution of the nonlinear Schrödinger equation

$$
U=u-2 P_{12}^{n-1}, \quad V=v+2 P_{21}^{n-1} .
$$

From the explicit expressions (4.1) and (4.2), it follows that all Bäcklund transformations are commutative ( functions $P_{12}^{n-1}, P_{21}^{n-1}$ are symmetrical to permutation of all pairs $\left.\lambda_{i}, \alpha_{i}\right)$.

If we choose the initial solution in a "zero" form $u=v^{*}=0, \quad g=\exp i 2(\lambda x+$ $\left.\lambda^{2} t\right) h$, we obtain an n-soliton solution in an explicit form. This solution of course coincides with the one obtained previously in [4][5] with the method of discrete transformation. 
The result of [4] corresponds to decomposition of the determinant of $2 n$th order into a sum of products of the corresponding minors $n$th order.

\section{Bäcklund Transformation in Its Original Form}

Bäcklund transformation, in contrast to the discrete one, is not local. Indeed, to obtain a new solution $U=V^{*}$ from a solution $u=v^{*}$, we have to resolve the L-A system (which is a system of linear differential equations), obtain the element $g$ and use its matrix elements to construct a new solution. This fact is related to the original Backlund's result of connecting the derivatives of the new and old solutions. We would like to show now how such relations can be obtained from the formalism of the previous section. Using the definition of $F\left(\lambda_{i}, \alpha_{i}\right)$ and taking into account equations of L-A pair (3.1), we obtain

$$
\begin{gathered}
F_{x}^{s} \equiv\left(F\left(\lambda_{s}, \alpha\right)_{s}\right)_{x}=i\left(v-2 \lambda_{s} F^{s}-u\left(F^{s}\right)^{2}\right), \\
F_{t}^{s}=i\left(\left(\lambda_{s} v+i \frac{v_{x}}{2}\right)-2\left(\lambda_{s}^{2}-\frac{u v}{2}\right) F^{s}-\left(\lambda_{s} u-i \frac{u_{x}}{2}\right)\left(F^{s}\right)^{2}\right) .
\end{gathered}
$$

Calculating $F^{1}, F^{2}$ via $2 B=u-U, 2 c=V-v$ from (3.10) and substituting the result into the system of derivatives above (with respect to $x$ or $t$ arguments), we obtain a system of two ordinary differential equations of the first order connecting $u, v$ and $U, V$ and containing parameters $\lambda_{1}=\lambda_{2}^{*}$ in explicit form. Two additional parameters $\alpha_{1}, \alpha_{2}=-\frac{1}{\alpha^{*}}$ have to arise in the process of integration of the last system in which $u=v^{*}$ considered as known and $U=V^{*}$ as unknown or visa versa.

\section{Second Automorphism $\sigma_{2}$ of the Nonlinear Schrödinger System}

Let us perform a change of variables $v=e^{i \theta}, u=e^{-i \theta}\left(R-\frac{i \theta_{x x}}{2}\right)$ in (2.2), where $R, \theta$ are two new unknown complex functions. We have

$$
2 \theta_{t}+\left(\theta_{x}\right)^{2}=2 R, \quad 2 R_{t}-\frac{1}{2} \theta_{x x x x}-2\left(\theta_{x} R\right)_{x}=0 .
$$

Of course the last system is invariant with obvious exchange $R \rightarrow R^{*}, \theta \rightarrow \theta^{*}$, which we call second inner automorphism $\sigma_{2}$ of nonlinear Schrödinger system. The system (6.1) after excluding $R$ is equivalent to a single equation

$$
\theta_{t t}-\frac{1}{4} \theta_{x x x x}+\left(\frac{3}{2} \theta_{x}^{2}+\theta_{t}\right) \theta_{x x}=0 .
$$

6.1. Method of Discrete Substitution. The nature of $\sigma_{2}$ automorphism consists in the fact that if discrete transformation (2.3) is interrupted on $2 n+1$ step (but not on $2 n$ one as it was in the case of $\sigma_{1}$ ), it has as its conclusion (6.2). Indeed in this case, in the middle of the lattice arises two solutions $\left(u_{n}=\frac{D_{n-1}}{D_{n}}, v_{n}=\frac{D_{n+1}}{D_{n}}\right)$ and $\left(u_{n+1}=\frac{D_{n}}{D_{n+1}}, v_{n+1}=\frac{D_{n+2}}{D_{n+1}}\right)$, which are connected by condition $v_{n}^{*}=u_{n+1}=$ $\frac{1}{v_{n}}, \quad u_{n}^{*}=v_{n+1}=v_{n}\left(u_{n} v_{n}+\left(\ln v_{n}\right)_{x x}\right)$, which lead to (6.1) and (6.2).

Using the technique of discrete transformation, n-soliton solution of the equation (6.2) and system (6.1) may be represented in terms of the following

$$
F=\sum_{k=1}^{2 n+1} c_{k} e^{i 2 L_{k}}, \quad f=\sum_{k=1}^{2 n+1} \frac{1}{c_{k}} e^{-i L_{k}} \frac{1}{\prod^{\prime}\left(\lambda_{k}-\lambda_{j}\right)^{2}}
$$

where $L_{i}=\lambda_{i}^{2} t+\lambda_{i} x$ and $\lambda_{i}, c_{i}-(2 n+1)$ numerical parameters connected via equation $F^{*}=f$. From the last condition, it follows the limitation on parameters 
of the problem: $2 s+1$ parameters $\lambda_{\beta}^{*}=\lambda_{\beta}$ are real ones, the remaining $2(n-s)$ are in complex congugated pairs $\lambda_{A}^{*}=\lambda_{B}(1 \leq A, B \leq(n-s))$. In all cases, $c_{i} c_{i}^{*}=\prod^{\prime}\left(\lambda_{i}-\lambda_{j}\right)^{2}$. Finally

$$
\theta=i \ln \frac{D_{n}}{D_{n+1}}, \quad R=\left(\ln \left(D_{n} D_{n}^{*}\right)\right)_{x x}
$$

where $D_{s}$ is the determinant of the s-order of the matrix:

$$
\left(\begin{array}{cccc}
F & F_{x} & F_{x x} & \cdots \\
F_{x} & F_{x x} & F_{x x x} & \cdots \\
F_{x x} & F_{x x x} & F_{x x x x} & \cdots
\end{array}\right) .
$$

6.2. Method of the Bäcklund Transformation. It is necessary to repeat word by word all calculations of the section 3 up to (3.8). The simplist solution of (6.1) is the following one soliton solution $\left(\theta=-2\left(\lambda_{0}^{2} t+\lambda_{0} x\right) \equiv-2 L_{0}, R=0\right)$. It is obvious that the corresponding group element $g$ belongs to the group of lower triangular matrices and may be represented in the following form:

$$
g(x, t: \lambda)=e^{\alpha X_{-}} e^{\tau H} .
$$

Taking into account equations of L-A pair (3.1), we obtain

$$
\begin{gathered}
g_{x} g^{-1}=\left(\alpha_{x}+2 \tau_{x} \alpha\right) X_{-}+\tau_{x} H=i\left(\lambda H+e^{-2 i L_{0}} X_{-}\right), \\
g_{t} g^{-1}=\left(\alpha_{t}+2 \tau_{t} \alpha\right) X_{-}+\tau_{t} H=i\left(\lambda^{2} H+e^{-2 i L_{0}}\left(\lambda+\lambda_{0}\right) X_{-}\right) .
\end{gathered}
$$

Solitions of the last equations are trivial with

$$
\alpha=\frac{1}{2} \frac{e^{-i 2 L_{0}}}{\lambda-\lambda_{0}}, \quad \tau=i\left(\lambda^{2} t+\lambda x\right)
$$

In the general case, let us represent element $g$ in the usual form of $S(2, C)$ group

$$
\begin{gathered}
g=e^{\alpha X_{+}} e^{\tau H} e^{\beta X_{-}}=\left(\begin{array}{ll}
e^{\tau}+\alpha \beta e^{-\tau} & \alpha e^{-\tau} \\
\beta e^{-\tau} & e^{-\tau} \\
\cdot &
\end{array}\right), \\
g^{\prime} g^{-1}=\left(\alpha^{\prime}-2 \tau^{\prime} \alpha-\alpha^{2} \beta^{\prime} e^{-2 \tau}\right) X_{+}+\left(\tau^{\prime}+\beta^{\prime} \alpha e^{-2 \tau}\right) H+\beta^{\prime} e^{-2 \tau} X_{-} .
\end{gathered}
$$

Taking into account equations of L-A pair (3.1), we obtain

$$
i \lambda=\left(\tau_{x}+\beta_{x} \alpha e^{-2 \tau}\right), \quad i v=\beta_{x} e^{-2 \tau} .
$$

From the last relation, we conclude that $\beta^{\prime} e^{-2 \tau}$ doesn't depend on $\lambda$ and $\alpha=$ $\frac{i \lambda-\tau_{x}}{\beta_{x} e^{-2 \tau}}$. Now let us rewrite the equation defining $C$ (3.5) using (6.3) (for clarity of notations, we change the parameters $\alpha_{1,2}$ in (3.9) to $\left.\nu_{1,2}\right)$

$$
\frac{1}{C}+\frac{1}{\lambda_{1}-\lambda_{2}}\left(\frac{e^{2 \tau_{1}}}{\beta_{1}+\nu_{1}}+\alpha_{1}-\frac{e^{2 \tau_{2}}}{\beta_{2}+\nu_{2}}-\alpha_{2}\right)=0 .
$$

The new solution $V=v+2 C(3.8)$ and the condition of its invariance with respect to $\sigma_{2}$, may be rewritten as $\left(v v^{*}=1\right.$ !)

$$
\frac{v}{C}+\frac{v^{*}}{C^{*}}=-2
$$


or using (6.5) we have $\left(i v=\beta_{x} e^{-2 \tau}\right)$

$$
\frac{v}{C}+\frac{\beta_{x} e^{-2 \tau}}{i\left(\lambda_{1}-\lambda_{2}\right)}\left(\frac{e^{2 \tau_{1}}}{\beta_{1}+\nu_{1}}+\alpha_{1}-\frac{e^{2 \tau_{2}}}{\beta_{2}+\nu_{2}}-\alpha_{2}\right)=0
$$

From $(6.6)\left(v^{*}=\frac{1}{v}\right)$, we obtain also

$$
v=C\left(-1+i \sqrt{\frac{1}{C C^{*}}-1}\right), \quad V=C\left(1+i \sqrt{\frac{1}{C C^{*}}-1}\right),
$$

which can be rewritten as

$$
\begin{gathered}
\frac{v}{C}+1+\frac{1}{i\left(\lambda_{1}-\lambda_{2}\right)}\left[\frac{\beta_{x}^{1}}{\left.\beta^{1}+\nu_{1}\right)}-\tau_{x}^{1}-\frac{\beta_{x}^{2}}{\left.\beta^{2}+\nu_{2}\right)}-\tau_{x}^{2}\right]=0, \\
\frac{v}{C}+1+\frac{1}{i\left(\lambda_{1}-\lambda_{2}\right)}\left[\ln \frac{\left(\beta^{1}+\nu_{1}\right) e^{-\tau_{1}}}{\left(\beta^{2}+\nu_{2}\right) e^{-\tau_{2}}}\right]_{x}=0 .
\end{gathered}
$$

Thus we have

$$
\frac{1}{i\left(\lambda_{1}-\lambda_{2}\right)}\left[\ln \frac{\left(\beta^{1}+\nu_{1}\right) e^{-\tau_{1}}}{\left(\beta^{2}+\nu_{2}\right) e^{-\tau_{2}}}\right]_{x}-\frac{1}{i\left(\lambda_{1}^{*}-\lambda_{2}^{*}\right)}\left[\ln \frac{\left(\beta^{1}+\nu_{1}\right) e^{-\tau_{1}}}{\left(\beta^{2}+\nu_{2}\right) e^{-\tau_{2}}}\right]_{x}^{*}=0 .
$$

Comparing (6.4) and L-A pair representation (3.1), we obtain

$$
\left(\alpha^{\prime}-2 \tau^{\prime} \alpha-\beta^{\prime} e^{-2 \tau}\right)=i u \equiv i \frac{1}{v} u v=i \frac{1}{v}\left(r-\frac{1}{2}(\ln v)_{x x}\right) .
$$

Substituting all relations obtained above, we have

$$
-r+\frac{i}{2} \theta_{x x}=\lambda^{2}-\tau_{x, x}+\left(\tau_{x}\right)^{2}+\left(\lambda+i \tau_{x}\right) \theta_{x} .
$$

In a L-A pair formalism, $\lambda$ has to be considered as a real parameter. Thus imaginary part of last equation has the form

$$
\theta_{x x}=\frac{1}{i}\left(\tau^{*}-\tau\right)_{x x}+\left(\tau^{*}+\tau\right)_{x} \theta_{x}+\left(\tau_{x}\right)^{2}-\left(\tau_{x}^{*}\right)^{2} .
$$

The last equation has an obvious first integral

$$
\theta_{x}-\frac{1}{i}\left(\tau^{*}-\tau\right)_{x}=c e^{\left(\tau^{*}+\tau\right)} .
$$

Noticing that $i e^{\theta}=\beta_{x} e^{-2 \tau}$ and substituting it into the last quation, we have

$$
\frac{1}{i}\left(\ln \beta_{x} e^{-\left(\tau^{*}+\tau\right)}\right)_{x}=c_{1} e^{\left(\tau^{*}+\tau\right)}, \quad \frac{1}{i}\left(\beta_{x} e^{-\left(\tau^{*}+\tau\right)}\right)_{x}=c_{1} \beta_{x} .
$$

Thus

$$
\frac{1}{i}\left(\beta_{x} e^{-\left(\tau^{*}+\tau\right)}\right)=e^{i \theta} e^{-\left(\tau^{*}-\tau\right)}=c_{1} \beta+c_{0} .
$$

Inverting (6.8), we obtain

$$
\beta+\nu=\frac{1}{c_{1}} e^{i \theta} e^{-\left(\tau^{*}-\tau\right)}+\nu-\frac{c_{0}}{c_{1}} .
$$

Putting the indices $k=1,2$ for $\lambda$ in the last expression, we have for the elements introduced in (6.7),

$$
\beta_{k}+\nu_{k}=\frac{1}{c_{1}^{k}} e^{i \theta} e^{-\left(\tau_{k}^{*}-\tau_{k}\right)}+\tilde{\nu}_{k},
$$


where $\tilde{\nu}_{k}=\nu_{k}-\frac{c_{0}^{k}}{c_{1}^{k}}$, and

$$
\beta_{k}^{*}+\nu_{k}^{*}=\frac{1}{\left(c_{1}^{k}\right)^{*}} e^{-i \theta} e^{\left(\tau_{k}^{*}-\tau_{k}\right)}+\tilde{\nu}_{k}^{*}
$$

After substituting the last expressions into (6.7) and simple manipulations, we arrive at two possibilities: First $\lambda_{1}=\lambda_{1}^{*}, \lambda_{2}=\lambda_{2}^{*}, \tilde{\nu}_{1} \tilde{\nu}_{1}^{*}=\frac{1}{c_{1}^{1}\left(c_{1}^{1}\right)^{*}}, \tilde{\nu}_{2} \tilde{\nu}_{2}^{*}=\frac{1}{c_{1}^{2}\left(c_{1}^{2}\right)^{*}}$, and second $\lambda_{1}=\lambda_{2}^{*}, \tilde{\nu}_{1} \tilde{\nu}_{2}^{*}=\frac{1}{c_{1}^{1}\left(c_{1}^{2}\right)^{*}}$. Of course, this results in the multisoliton solutions which coincide with the ones obtained above by the method of discrete substitution.

\section{Outlook}

First, we would like to explain why, in spite of our comments in the Introduction, Bäcklund transformations are interesting objects for investigation. Bäcklund transformations, as well as discrete ones, are canonical transformations. This means that under these transformations, densities of conserved quantities change by complete derivatives. In the case we considered above this applies in particular to the energy density. Therefore, if the energy density of the initial solution is $e$, after the application of the Backlund transformation it will be $e_{B}=e+\Delta_{x}$. The term $\Delta$ contains all parameters $\lambda_{1}=\lambda_{2}^{*}, \alpha_{1}=-\frac{1}{\alpha^{*}}$, which define the Bäcklund transformation. For obtaining an explicit expression for $\Delta$ an additional (not a very cumbersome) calculation, using the technique of [4][5], is necessary. If $e$ itself has been obtained from some other solution and so on, we will have a final expression

$$
e_{n}=\sum \Delta_{x}^{i}
$$

Each $\Delta^{i}$ contains only parameters of previous transformations. Because the total energy for an $n$-soliton solution is equal to $\mathrm{n}$, it is very natural to assume that all terms of the last sum have the same behavior at infinity and contribute 1 to the total energy after integration.

It is completely obvious that $\sigma_{1}$ and $\sigma_{2}$ exist for all integrable systems possessing soliton solutions and a discrete transformation. Indeed, as it was explained above, this is related only to the parity of the step, odd $(2 n)$ or even $(2 n+1)$, on which the discrete transformation applied to the initial solution is interrupted. Without any doubts, all results of this paper also apply to supersymmetric integrable hierarchies $[\mathbf{6}]$ and multicomponent matrix-type hierarchies $[\mathbf{7}],[\mathbf{8}]$. What are the systems connected to $\sigma_{2}$ is completely unknown to the author at this moment.

Acknowledgements: The author is greatly indebted to E. A. Yuzbajan for discussions of the results and helps in preparing the manuscript for publication. 


\section{Appendix}

Let us rewrite (3.1) and (3.2) in the equivalent form

$$
\begin{aligned}
& \left(\begin{array}{cc}
A_{x} & B_{x} \\
C_{x} & D_{x}
\end{array}\right)+\left(\begin{array}{lr}
\lambda+A & B \\
C & \lambda+D
\end{array}\right) i\left(\begin{array}{rr}
\lambda, & u \\
v & -\lambda
\end{array}\right) \\
& =i\left(\begin{array}{cc}
\lambda, & U \\
V & -\lambda
\end{array}\right)\left(\begin{array}{lr}
\lambda+A & B \\
C & \lambda+D
\end{array}\right) \\
& \left(\begin{array}{cc}
A_{t} & B_{t} \\
C_{t} & D_{t}
\end{array}\right)+\left(\begin{array}{cc}
\lambda+A & B \\
C & \lambda+D
\end{array}\right) i\left(\begin{array}{rr}
\lambda^{2}-\frac{u v}{2}, & \lambda u-i \frac{u_{x}}{2} \\
\lambda v+i \frac{v_{x}}{2} & -\lambda^{2}+\frac{u v}{2}
\end{array}\right) \\
& =i\left(\begin{array}{cc}
\lambda^{2}-\frac{U V}{2}, & \lambda U-i \frac{U_{x}}{2} \\
\lambda V+i \frac{V_{x}}{2} & -\lambda^{2}+\frac{U V}{2}
\end{array}\right)\left(\begin{array}{lr}
\lambda+A & B \\
C & \lambda+D
\end{array}\right) .
\end{aligned}
$$

From which we have the following system of equations

$$
\begin{aligned}
& u-U=2 B, \quad v-V=-2 C, \quad i A_{x}=B v-C U, \\
& i D_{x}=C u-B V, \quad i B_{x}=A u-D U, \quad i C_{x}=D v-A V
\end{aligned}
$$

and the same kind of the equations with respect to $t$ differentiation. As a result we have $A+D, A D-B C$ doesn't depend on $x, t$ arguments and thus is representable in the form $A+D=\lambda_{1}+\lambda_{2}, A D-B C=\lambda_{1} \lambda_{2}$. Besides, functions $C$ and $D$ satisfy the following system of equations

$$
\begin{gathered}
-i C_{t}-\frac{1}{2}\left(C_{x x}+4 i k C_{x}+4 k^{2} C\right)-C(B C)+\frac{\left(C_{x}\right)^{2} B}{4(\epsilon-C B)}=0, \\
i B_{t}-\frac{1}{2}\left(B_{x x}-4 i k B_{x}+4 k^{2} B\right)-B(B C)+\frac{\left(B_{x}\right)^{2} C}{4(\epsilon-C B)}=0,
\end{gathered}
$$

where $k=\lambda_{1}+\lambda_{2}, \epsilon=\left(\frac{\left.\lambda_{1}-\lambda_{2}\right)}{2}\right)^{2}$. The last system of course is equivalent to nonlinear Schrödinger equation. More other solutions of (2.2) are connected with the solution of the above system via relations

$$
v=-C-\frac{i C_{x}+k C}{2 \sqrt{\epsilon-B C}}, \quad u=B+\frac{i B_{x}-k C}{2 \sqrt{\epsilon-B C}} .
$$

\section{References}

1. A. N. Leznov, the method of scalar $L-A$ pair and the soliton solutions of the periodic Toda lattice, Proceedings of II International Workshop: "Nonlinear and Turbulent Process", Kiev 1983, Gordon and Beach, New York, 1984, 1437.

A. N. Leznov, Functional Anal. Appl. 18 (1984), 83.

A. N. Leznov, Lett. Math. Phys. 8, N5 (1984), 379.

A. N. Leznov, Lett. Math. Phys. 8, N4 (1984), 353.

2. A. N. Leznov and A. V. Razumov, J. Math. Phys. 35 (1994), 4067.

3. A. N. Leznov and A. V. Razumov, J. Math. Phys. 35 (1994), 1738.

4. A. N. Leznov, Completely integrable systems, IHEP 92-112 (1992), 1.

5. A. N. Leznov, Physics of Elementary Particals and Atom Nuclears 27, v.5 (1996), 1161.

6. A. N. Leznov and A. S. Sorin, Phys. Lett. B389 (1996), 494.

7. A. N. Leznov and E. A. Yusbashyan, LMP 35 (1995), 345.

8. A. N. Leznov and E. A. Yusbashyan, Nucl.Phys. B 496,(3) (1997), 643.

9. L.D.Fadeev and L.A.Takhtajan, Hamiltonian Approach in Soliton Theory, Moscow, Nauka (1985).

Universidad Autonoma del Estado de Morelos, CiICAP, Cuernavaca, Mexico

E-mail address: andrey@uaem.mx 